\title{
Pharmacokinetic Properties of Fast-Acting Insulin Aspart Administered in Different Subcutaneous Injection Regions
}

\author{
Ulrike Hövelmann $^{1} \cdot$ Tim Heise $^{1} \cdot$ Leszek Nosek $^{1} \cdot$ Bettina Sassenfeld $^{1}$. \\ Karen Margrete Due Thomsen ${ }^{2} \cdot$ Hanne Haahr $^{3}$
}

Published online: 10 February 2017

(c) The Author(s) 2017. This article is published with open access at Springerlink.com

\begin{abstract}
Background Fast-acting insulin aspart (faster aspart) is insulin aspart set in a new formulation with faster initial absorption after subcutaneous administration. This study investigated the pharmacokinetic properties, including the absolute bioavailability, of faster aspart when administered subcutaneously in the abdomen, upper arm or thigh.

Methods In a randomised, open-label, crossover trial, 21 healthy male subjects received a single injection of faster aspart at five dosing visits: $0.2 \mathrm{U} / \mathrm{kg}$ subcutaneously in the abdomen, upper arm and thigh, intramuscularly in the thigh and $0.02 \mathrm{U} / \mathrm{kg}$ intravenously. Blood sampling for pharmacokinetics was performed pre-dose and frequently thereafter until $12 \mathrm{~h}$ post-dose $(8 \mathrm{~h}$ after intravenous administration).

Results Onset of appearance ( $\sim 3 \mathrm{~min})$, time to $50 \%$ of maximum concentration ( $t_{\text {Early }} 50 \% \mathrm{Cmax} ; \sim 20 \mathrm{~min}$ ) and time to maximum concentration $\left(t_{\max } ; \sim 55 \mathrm{~min}\right.$ ) were all similar between injection regions. Early exposure within the first $2 \mathrm{~h}$ after injection $\left(\mathrm{AUC}_{\mathrm{IAsp}, 0-1 \mathrm{~h}}\right.$ and $\mathrm{AUC}_{\mathrm{IAsp}, 0-2 \mathrm{~h}}$ ) as well as maximum concentration $\left(C_{\max }\right)$ were comparable for the abdomen and upper arm, but were $\sim 25 \%$ lower for the thigh as seen previously for other mealtime insulin products. Total exposure $\left(\mathrm{AUC}_{\mathrm{IAsp}, 0-\mathrm{t}}\right)$ was similar for the abdomen, upper arm and thigh, and absolute bioavailability
\end{abstract}

Tim Heise

tim.heise@profil.com

1 Profil Institut für Stoffwechselforschung $\mathrm{GmbH}$, Hellersbergstrasse 9, 41460 Neuss, Germany

2 Novo Nordisk, Alfred Nobels Vej 27, 9220 Aalborg Ø, Denmark

3 Novo Nordisk, Vandtårnsvej 114, 2860 Søborg, Denmark was $\sim 80 \%$ after subcutaneous administration of faster aspart in all three injection regions.

Conclusion The current study supports the ultra-fast pharmacokinetic characteristics of faster aspart across different injection regions, with administration in the abdomen and upper arm resulting in greater early exposure than in the thigh.

ClinicalTrials.gov identifier: NCT02089451.

\section{Key Points}

Fast-acting insulin aspart has an ultra-fast onset of exposure independent of injection region (abdomen, upper arm or thigh).

As previously observed for other mealtime insulin products, early exposure ( $<2 \mathrm{~h}$ after administration) and maximum concentration of fast-acting insulin aspart are lower for the thigh than for the abdomen and upper arm, while total exposure is comparable between all three injection regions (absolute bioavailability of $\sim 80 \%$ ).

Fast-acting insulin aspart may be administered subcutaneously in the abdomen, upper arm or thigh; however, administration in the abdomen and upper arm leads to the fastest pharmacokinetic profile.

\section{Introduction}

For practical, physiological and anatomical reasons, the preferred body region for subcutaneous injection of insulin varies among patients with diabetes [1]. Recommended 
injection regions include the abdomen, thigh, upper arm and buttock [2]. It is well recognised that the rate and/or extent of insulin absorption may differ between injection regions [3-7], e.g. slower absorption for the thigh versus the abdomen is a consistent finding $[4,5,7]$. Therefore, it is important for any new insulin that its pharmacokinetic properties are characterised when administered in different anatomical regions of the body.

Fast-acting insulin aspart (faster aspart) is insulin aspart (IAsp) designed in a new formulation including two additional excipients (L-arginine and niacinamide), serving to achieve a stable formulation that conveys earlier onset of appearance and faster initial absorption rate after subcutaneous injection. Faster aspart has twice-as-fast onset of appearance and two-fold higher early insulin exposure leading to faster onset of action and more than $50 \%$ greater early glucose-lowering effect compared with IAsp [8-10]. Consequently, use of faster aspart may lead to better postprandial glucose control relative to current rapid-acting insulins.

The current study aimed to investigate the pharmacokinetics, including the absolute bioavailability, of faster aspart administered subcutaneously in the abdomen, upper arm or thigh in healthy subjects.

\section{Methods}

\subsection{Trial Design and Participants}

This randomised, open-label, five-period, crossover trial was approved by Bundesinstitut für Arzneimittel und Medizinprodukte (the local health authority) and by Ärztekammer Nordrhein (an independent ethics committee), conducted according to the Declaration of Helsinki, Good Clinical Practice and guidelines on bioavailability trials [11, 12], and registered at ClinicalTrials.gov (NCT02089451). Informed consent was obtained from all individual participants included in the study.

Eligible subjects were healthy men and women (however, all enrolled subjects were men) aged 18-64 years, with a body mass index of $20.0-28.0 \mathrm{~kg} / \mathrm{m}^{2}$ and fasting plasma glucose $\leq 5.6 \mathrm{mmol} / \mathrm{L}$. Individuals with abnormal clinical laboratory results, those using prescription or nonprescription drugs (except topical medication, oral contraceptives, routine vitamins and occasional use of ibuprofen and paracetamol), smokers and pregnant or breastfeeding women were excluded.

\subsection{Procedures}

The visit structure is shown in Fig. 1. Subjects received faster aspart single-dosing at five visits: $0.2 \mathrm{U} / \mathrm{kg}$ subcutaneously in the abdomen (lifted skin fold of the lower abdominal wall above the inguinal area), upper arm (lifted skin fold of the outer aspect of the upper arm) and thigh (lifted skin fold of the anterior surface of the thigh), $0.2 \mathrm{U} / \mathrm{kg}$ intramuscularly in the thigh and $0.02 \mathrm{U} / \mathrm{kg}$ intravenously (1-min injection through a catheter inserted into a hand or forearm vein). Faster aspart $(100 \mathrm{U} / \mathrm{mL}$; Novo Nordisk, Bagsværd, Denmark) was provided in a PDS290 pen-injector prefilled pen (Novo Nordisk) for subcutaneous administration and in a $3-\mathrm{mL}$ Penfill ${ }^{\circledR}$ cartridge for intramuscular and intravenous administration.

At the dosing visits, subjects came to the clinic in the morning after an overnight fast and received faster aspart. For safety reasons, to maintain blood glucose within the normal range, a euglycaemic clamp (ClampArt ${ }^{\circledR}$; Profil, Neuss, Germany) was performed [9]. The clamp target was $4.5 \mathrm{mmol} / \mathrm{L}$ and clamp duration was up to $12 \mathrm{~h}$ post-dosing (8 $\mathrm{h}$ after intravenous administration).

For subcutaneous and intramuscular administration, blood was sampled for pharmacokinetics within 2 min predose, then every 2 min from dosing until 20 min post-dose, every 5 min until $80 \mathrm{~min}$, every $10 \mathrm{~min}$ until $2 \mathrm{~h}$, every 15 min until $3 \mathrm{~h}$, and then at 3.5, 4, 5, 6, 7, 8, 10 and $12 \mathrm{~h}$ postdose. For intravenous administration, the same schedule applied until $2.5 \mathrm{~h}$, followed by sampling at 3, 4, 6 and $8 \mathrm{~h}$ post-dose. Free (unbound) serum IAsp concentration was assessed by polyethylene glycol precipitation using an IAsp-specific enzyme-linked immunosorbent assay [lower limit of quantification (LLOQ) of $10 \mathrm{pmol} / \mathrm{L}$ ] validated according to relevant guidelines [13, 14]. Safety assessments included adverse events, local tolerability at the injection site, hypoglycaemic episodes (defined as 'confirmed' when they were either 'severe' according to the American Diabetes Association [15] or verified by plasma glucose $<3.1 \mathrm{mmol} / \mathrm{L})$, laboratory safety parameters, physical examination, vital signs and electrocardiogram.

\subsection{Statistical Analyses}

Statistical analysis was performed using SAS version 9.3 (SAS Institute, Cary, NC, USA).

Assuming a residual standard deviation for the primary endpoint (total exposure; $\mathrm{AUC}_{\mathrm{IAsp}, 0-\mathrm{t}}$ ) of 0.25 [8], 18 completers would expectedly yield a $95 \%$ confidence interval (CI) of (0.85-1.18) for the geometric mean ratio of $\mathrm{AUC}_{\mathrm{IAsp}, 0-\mathrm{t}}$ between any two subcutaneous injection regions, if the observed ratio was 1.00 . This was considered sufficiently narrow to support the primary objective of comparing total exposure between administration in the abdomen, upper arm and thigh.

Endpoints related to onset of exposure included onset of appearance (time from faster aspart administration until the first time of insulin concentration $\geq$ LLOQ), time to $50 \%$ of 


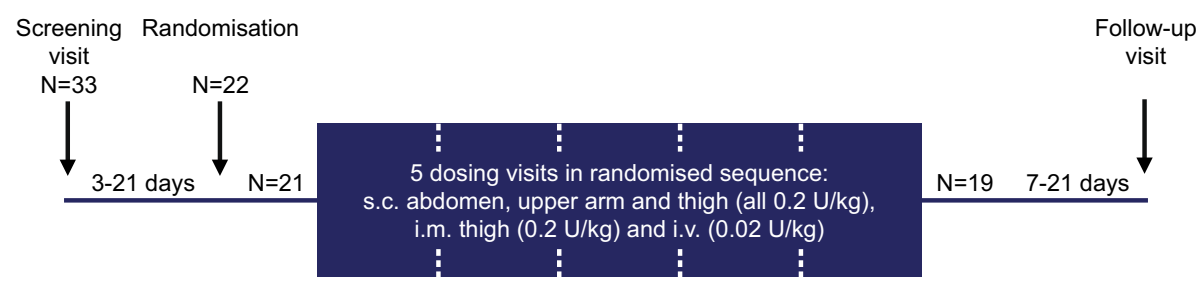

Fig. 1 Study design and subject disposition. Each subject participated in a total of five dosing visits in randomised sequence. All dosing visits were separated by a washout period of 3-12 days. The three randomised subjects who did not complete the trial were all due to withdrawal of consent (one before first dosing, one after

maximum concentration ( $t_{\text {Early } 50 \%}$ Cmax $)$ and time to maximum concentration $\left(t_{\max }\right)$. Endpoints to evaluate early exposure were areas under the curve (AUC) for IAsp from 0 to $1 \mathrm{~h}\left(\mathrm{AUC}_{\mathrm{IAsp}, 0-1 \mathrm{~h}}\right)$ and 0 to $2 \mathrm{~h}\left(\mathrm{AUC}_{\mathrm{IAsp}, 0-2 \mathrm{~h}}\right)$. Overall exposure was assessed by $\mathrm{AUC}_{\mathrm{IAsp}, 0-\mathrm{t}}$, maximum concentration $\left(C_{\max }\right)$ and the IAsp AUC from 0 to infinity $\left(\mathrm{AUC}_{\mathrm{IAsp}, 0-\infty}\right)$.

For determination of onset of appearance and AUC endpoints after subcutaneous administration, fitted curves based on compartmental modelling were used from the time of administration until the first quantifiable concentration, as previously described [9]. After intravenous administration, the initial part of the AUC was derived using back-extrapolation to $1 \mathrm{~min}$ (end of intravenous injection) and setting the concentration to zero at $t=0$ (while excluding the 2-min sample since appropriate distribution of trial product had not been achieved at this time point). Between the first and last quantifiable concentration, AUCs were calculated using the linear trapezoidal technique. After the last quantifiable concentration, extrapolation based on the terminal slope was applied until the last pharmacokinetic sampling time point (for $\mathrm{AUC}_{\mathrm{IAsp}, 0-\mathrm{t}}$ ) or until infinity (for $\left.\mathrm{AUC}_{\mathrm{IAsp}, 0-\infty}\right)$.

Onset and early exposure endpoints were compared between subcutaneous injection regions using descriptive statistics. AUC $\mathrm{IAsp}, 0-\mathrm{t}_{\mathrm{I}}$ and $C_{\max }$ were log-transformed and compared between subcutaneous injection regions in an analysis of variance with treatment and period as fixed effects and subject as random effect. A covariance model allowing for different variances, but identical correlation between treatments for each subject, was used to account for heteroscedasticity. In order to assess the absolute bioavailability of faster aspart after subcutaneous administration, $\mathrm{AUC}_{\mathrm{IAsp}, 0-\infty}$ was log-transformed and analysed using the same model as for $\mathrm{AUC}_{\mathrm{IAsp}, 0-\mathrm{t}}$ and $C_{\text {max }}$. The estimated treatment ratios and $95 \% \mathrm{CIs}$ for $\mathrm{AUC}_{\mathrm{IAsp}, 0-\infty}$ were calculated between each of the subcutaneous injection regions and intravenous administration (after dose-adjustment to $0.2 \mathrm{U} / \mathrm{kg}$ for intravenous administration). subcutaneous administration in the thigh and one after intravenous administration and subcutaneous administration in the abdomen and upper arm). I.m. intramuscularly, i.v. intravenously, $N$ number of subjects, s.c. subcutaneously

\section{Results}

\subsection{Subjects}

Of 33 subjects screened, 22 were randomised, 21 were exposed to faster aspart and 19 subjects completed the trial (Fig. 1). Subject characteristics are shown in Table 1.

\subsection{Pharmacokinetics}

Observed pharmacokinetic time-profiles for subcutaneously administered faster aspart are shown in Fig. 2. Onset of appearance, $t_{\text {Early } 50 \% \mathrm{Cmax}}$ and $t_{\max }$ were all similar between injection regions (Table 2). $\mathrm{AUC}_{\mathrm{IAsp}, 0-1 \mathrm{~h}}$ and $\mathrm{AUC}_{\mathrm{IAsp}, 0-2 \mathrm{~h}}$ were comparable for the abdomen and upper arm, but lower for the thigh.

Total exposure $\left(\mathrm{AUC}_{\mathrm{IAsp}, 0-\mathrm{t}}\right)$ was similar for all three injection regions, while $C_{\max }$ was comparable between the abdomen and upper arm but lower for the thigh (Table 3).

Treatment ratios $(95 \% \mathrm{CI})$ for $\mathrm{AUC}_{\mathrm{IAsp}, 0-\infty}$ for the abdomen, upper arm and thigh versus intravenous administration were $0.83(0.74-0.93), 0.77(0.68-0.87)$ and 0.77

Table 1 Subject characteristics $(N=21)$

\begin{tabular}{lc}
\hline Characteristic & Value \\
\hline Age, years & $32.8(7.7)$ \\
Sex & \\
Female, $N(\%)$ & $0(0.0)$ \\
Male, $N(\%)$ & $21(100.0)$ \\
Race & \\
White, $N(\%)$ & $20(95.2)$ \\
Other ${ }^{\mathrm{a}}, N(\%)$ & $1(4.8)$ \\
Height, m & $1.82(0.07)$ \\
Body weight, kg & $84.0(7.7)$ \\
BMI, kg/m & \\
Fasting plasma glucose, mmol/L & $25.4(1.8)$ \\
\hline
\end{tabular}

Data are mean (standard deviation) unless otherwise stated $B M I$ body mass index, $N$ number of subjects

a Mixed Asian and White 


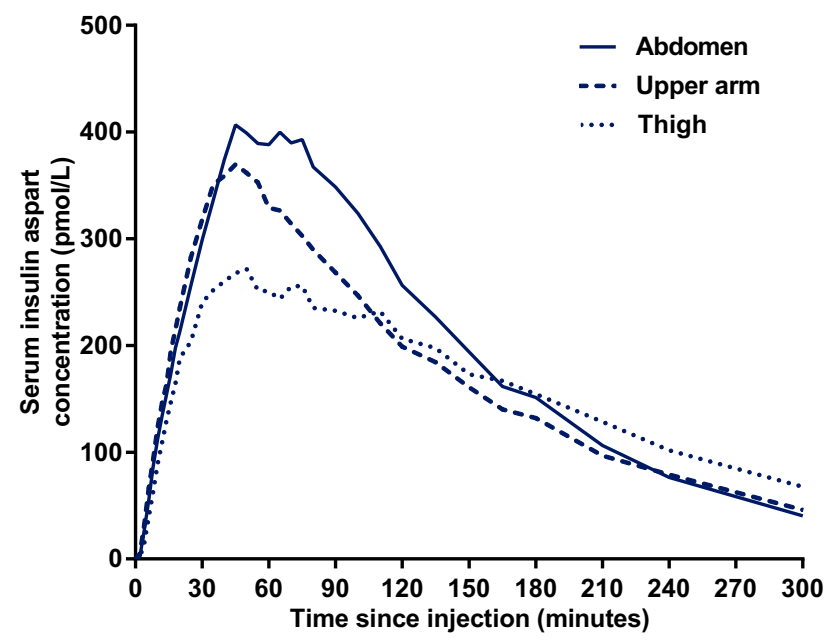

Fig. 2 Mean observed serum insulin aspart concentration-time profiles for $0.2 \mathrm{U} / \mathrm{kg}$ faster aspart administered subcutaneously in the abdomen, upper arm or thigh

(0.68-0.87), respectively. Thus, absolute bioavailability of faster aspart was close to $80 \%$ after subcutaneous administration in all three injection regions (abdomen 83\%; upper arm $77 \%$; thigh $77 \%$ ).

Intramuscular administration of faster aspart resulted in a median onset of appearance of 2.6 min (minimummaximum: $1.1-5.3), t_{\text {Early } 50 \% ~ C \max }$ of $14.0 \mathrm{~min}$ (10.0-35.0) and $t_{\max }$ of $45.0 \mathrm{~min}$ (25.0-90.0), mean \pm standard deviation $\mathrm{AUC}_{\mathrm{IAsp}, 0-1 \mathrm{~h}}$ and $\mathrm{AUC}_{\mathrm{IAsp}, 0-2 \mathrm{~h}}$ of $198.9 \pm 80.0$ and $414.1 \pm 141.9 \mathrm{pmol} \cdot \mathrm{h} / \mathrm{L}$, and least square mean total exposure of $696.7 \mathrm{pmol} \cdot \mathrm{h} / \mathrm{L}$ and $C_{\max }$ of $270.1 \mathrm{pmol} / \mathrm{L}$.

\subsection{Safety}

Faster aspart was well tolerated with no safety issues identified during the trial. A total of 11 adverse events (AEs) were reported (10 mild and 1 moderate). All were non-serious and no obvious pattern occurred across injection region or route of administration. Headache was the most frequently reported AE (5 events). There were no clinically significant findings in safety laboratory parameters, vital signs, physical examination or electrocardiogram. No confirmed hypoglycaemic episodes or injection site reactions were reported.

\section{Discussion}

Among the key pharmacological properties of faster aspart are its faster onset of exposure and glucoselowering effect versus IAsp [8-10]. It was therefore reassuring in the present study that onset of exposure for faster aspart was unaffected by subcutaneous injection region. Total exposure for faster aspart was comparable between injection regions, as previously seen for insulin lispro, insulin glulisine and IAsp (Table 4). Pharmacodynamics were not assessed in the current study. However, a robust correlation between pharmacokinetics and pharmacodynamics was recently shown for faster aspart [9], suggesting the present pharmacokinetic results may likely translate to similar pharmacodynamics for different injection regions.

The lower early exposure and $C_{\max }$ of faster aspart for the thigh compared with the abdomen was expected, since a similar difference was shown in several previous studies with regular human insulin [3-6] and with rapid-acting insulin analogues (Table 4). For mealtime insulins, early exposure and $C_{\max }$ are important factors determining the ability to control postprandial glucose. Therefore, it is expected for faster aspart, and for other mealtime insulins, that abdominal administration is better able to reduce postprandial glucose compared with administration in the thigh.
Table 2 Onset of exposure and early exposure for $0.2 \mathrm{U} / \mathrm{kg}$ faster aspart administered subcutaneously in the abdomen, upper arm or thigh $(N=21)$

\begin{tabular}{llll}
\hline & Abdomen & Upper arm & Thigh \\
\hline Onset of exposure & & & \\
$\quad$ Onset of appearance $(\min )$ & $2.8(1.3-5.0)$ & $2.3(1.1-5.3)$ & $3.4(1.8-5.9)$ \\
$t_{\text {Early } 50 \% \text { Cmax }}(\min )$ & $25.0(12.0-35.0)$ & $18.0(8.0-30.0)$ & $20.0(12.0-40.0)^{\mathrm{a}}$ \\
$\quad t_{\max }(\min )$ & $55.0(30.0-100.0)$ & $50.0(30.0-100.0)$ & $57.5(20.0-210.0)$ \\
Early exposure & & & \\
AUC $_{\text {IAsp }, 0-1 \mathrm{~h}}(\mathrm{pmol} \cdot \mathrm{h} / \mathrm{L})$ & $265.1 \pm 121.5$ & $261.6 \pm 136.2$ & $192.4 \pm 114.2$ \\
AUC $_{\text {IAsp }, 0-2 \mathrm{~h}}(\mathrm{pmol} \cdot \mathrm{h} / \mathrm{L})$ & $607.9 \pm 259.0$ & $529.4 \pm 213.9$ & $426.3 \pm 174.1$ \\
\hline
\end{tabular}

Data for onset of exposure endpoints are median (minimum-maximum) and data for early exposure endpoints are mean \pm standard deviation

AUC area under the curve, IAsp insulin aspart, $N$ number of subjects, $t_{\text {Early }} 50 \%$ Cmax time to $50 \%$ of maximum insulin aspart concentration in the early part of the pharmacokinetic profile, $t_{\max }$ time to maximum insulin aspart concentration

a Comparison with abdomen and upper arm should be interpreted with caution due to the maximum concentration being lower for thigh compared to abdomen and upper arm (see Table 3) 
Table 3 Total exposure and maximum concentration for 0.2 $\mathrm{U} / \mathrm{kg}$ faster aspart administered subcutaneously in the abdomen, upper arm or thigh $(n=21)$
Table 4 Differences between subcutaneous injection regions for total exposure and maximum concentration of insulin lispro, insulin glulisine, insulin aspart and faster aspart

\begin{tabular}{lrll}
\hline & LS mean & Treatment ratio $(95 \% \mathrm{CI})$ & $P$ value \\
\hline Total exposure $\left(\mathrm{AUC}_{\mathrm{IAsp}, 0-\mathrm{t}}\right)(\mathrm{pmol} \cdot \mathrm{h} / \mathrm{L})$ & 1000.9 & & \\
Abdomen & 921.9 & & \\
Upper arm & 926.5 & & 0.070 \\
Thigh & & $0.92(0.84-1.01)$ & 0.092 \\
Upper arm/abdomen & & $0.93(0.85-1.01)$ & 0.907 \\
Thigh/abdomen & & $1.00(0.92-1.09)$ & \\
Thigh/upper arm & 394.6 & & \\
Maximum concentration $\left(C_{\text {max }}\right)(\mathrm{pmol} / \mathrm{L})$ & & \\
Abdomen & 363.8 & & 0.447 \\
Upper arm & 275.7 & $0.92(0.74-1.14)$ & 0.002 \\
Thigh & & $0.70(0.56-0.87)$ & 0.016 \\
Upper arm/abdomen & & $0.76(0.61-0.95)$ & \\
Thigh/abdomen & & & \\
Thigh/upper arm & & & \\
\hline
\end{tabular}

$A U C$ area under the curve, $C I$ confidence interval, $C_{\max }$ maximum concentration, IAsp insulin aspart, $L S$ Mean least square mean, $N$ number of subjects

\begin{tabular}{|c|c|c|c|c|c|}
\hline & \multicolumn{3}{|l|}{ Mean $^{\mathrm{a}}$} & \multicolumn{2}{|l|}{ Ratio (\%) } \\
\hline & Abdomen & Upper arm & Thigh & Upper arm/abdomen & Thigh/abdomen \\
\hline \multicolumn{6}{|c|}{ Total exposure $(\mathrm{pmol} \cdot \mathrm{h} / \mathrm{L})$} \\
\hline Insulin lispro [5] & 1388 & 1313 & 1277 & 95 & 92 \\
\hline Insulin glulisine [7] & 2182 & 2119 & 2021 & 97 & 93 \\
\hline Insulin aspart [6] & 1300 & 1361 & 1265 & 105 & 97 \\
\hline Faster aspart & $1001^{\mathrm{b}}$ & $922^{\mathrm{b}}$ & $927^{\mathrm{b}}$ & 92 & 93 \\
\hline \multicolumn{6}{|c|}{ Maximum concentration $(\mathrm{pmol} / \mathrm{L})$} \\
\hline Insulin lispro [5] & 589 & 395 & 458 & 67 & 78 \\
\hline Insulin glulisine [7] & 1003 & 821 & 684 & 82 & 68 \\
\hline Insulin aspart [6] & 501 & 506 & 422 & 101 & 84 \\
\hline Faster aspart & $395^{\mathrm{b}}$ & $364^{\mathrm{b}}$ & $276^{\mathrm{b}}$ & 92 & 70 \\
\hline
\end{tabular}

All data are for a dose of $0.2 \mathrm{U} / \mathrm{kg}$. Insulin glulisine data have been dose-adjusted from $0.1 \mathrm{U} / \mathrm{kg}$ assuming dose-proportionality

a Absolute levels of total exposure and maximum concentration should be compared with caution between the different insulin products, since results originate from different studies

b Absolute levels of total exposure and maximum concentration for faster aspart are not comparable to the other insulin products, since free (i.e. unbound), rather than total (i.e. bound plus unbound), insulin concentration was measured in the current study
Early exposure and $C_{\max }$ for faster aspart were comparable for the abdomen versus the upper arm (Tables 2 and $3)$. The same was observed for $C_{\max }$ of IAsp in a previous study [6] (Table 4). In contrast, for insulin lispro and insulin glulisine, $C_{\max }$ appears lower for the upper arm versus the abdomen (Table 4). Moreover, for insulin glulisine, early exposure within $1 \mathrm{~h}$ after administration is $\sim 20 \%$ lower for the upper arm than for the abdomen (and $\sim 40 \%$ lower for the thigh than for the abdomen) [7]. These findings imply that faster aspart may be administered in the abdomen or upper arm with no difference in its ultra-fast pharmacokinetic properties. In contrast, for insulin lispro and insulin glulisine, some difference in early pharmacokinetic response, and thereby in postprandial glucoselowering effect, may be expected for administration in the abdomen versus the upper arm. Differences in insulin absorption rate between injection regions are due to subcutaneous anthropometry and blood flow [16]. It is speculated that faster aspart absorption is less prone to variations in these factors for some yet unidentified reason.

One limitation of the current study was that pharmacodynamics were not assessed. In line with regulatory 
guidance, healthy subjects were included in the current trial $[11,12]$. Consequently, pharmacodynamics would presumably have been affected by endogenous insulin secretion of the healthy subjects. It was therefore decided prospectively to include a glucose clamp only for safety precaution. Another limitation was that all subjects participating in the present study were males (although both males and females were eligible). A third limitation was related to the interpretation of $t_{\text {Early } 50 \% \text { Cmax }}$ after administration in the thigh relative to the abdomen and upper arm, which should be made with caution since $C_{\max }$ was lower for the thigh versus the abdomen and upper arm [17].

Intramuscular administration in the thigh was only included for regulatory purposes. Compared with subcutaneous administration in the thigh, intramuscular administration resulted in similar onset of appearance, shorter $t_{\text {Early } 50 \% \text { Cmax }}$, shorter $t_{\max }$, similar early exposure and $C_{\max }$, and lower total exposure. The clinical relevance is, however, assessed to be somewhat limited as faster aspart will not be indicated for intramuscular administration.

\section{Conclusion}

This study showed similar ultra-fast onset of exposure of faster aspart after subcutaneous administration in the abdomen, upper arm and thigh. As previously observed for other mealtime insulin products, early exposure and maximum concentration were lower for the thigh than for the abdomen and upper arm. The current study supports the option of administering faster aspart in the abdomen, upper arm or thigh, with the abdomen and upper arm providing greater early exposure and, hence, taking full advantage of the more rapid pharmacokinetic properties of this new formulation.

Acknowledgements The authors are grateful to Alexandru L. Dinita, MD, Novo Nordisk, for reviewing and providing input to the manuscript and Carsten Roepstorff, PhD, CR Pharma Consult, Copenhagen, Denmark for contributing with medical writing support, which was funded by Novo Nordisk.

\section{Compliance with Ethical Standards}

All procedures performed in studies involving human participants were in accordance with the ethical standards of the institutional and/ or national research committee and with the 1964 Helsinki declaration and its later amendments or comparable ethical standards. Informed consent was obtained from all individual participants included in the study.

Funding This study was funded by Novo Nordisk.

Conflict of interest Tim Heise is a shareholder in Profil, which received research funds from Adocia, AstraZeneca, Becton Dickinson, Biocon, Boehringer Ingelheim, Dance Biopharm, Eli Lilly, Grünenthal, Gulf Pharmaceutical Industries, Johnson \& Johnson,
Marvel, MedImmune, Medtronic, Novartis, Novo Nordisk, Roche Diagnostics, Sanofi, Senseonics and Zealand Pharma. In addition, Tim Heise is a member of advisory panels for Novo Nordisk and received speaker honoraria and travel grants from Eli Lilly, Mylan and Novo Nordisk. Karen Margrete Due Thomsen and Hanne Haahr are employees and shareholders of Novo Nordisk. Ulrike Hövelmann, Leszek Nosek and Bettina Sassenfeld declare no conflicts of interest.

Open Access This article is distributed under the terms of the Creative Commons Attribution-NonCommercial 4.0 International License (http://creativecommons.org/licenses/by-nc/4.0/), which permits any noncommercial use, distribution, and reproduction in any medium, provided you give appropriate credit to the original author(s) and the source, provide a link to the Creative Commons license, and indicate if changes were made.

\section{References}

1. De Coninck C, Frid A, Gaspar R, et al. Results and analysis of the 2008-2009 Insulin Injection Technique Questionnaire survey. J Diabetes. 2010;2:168-79.

2. Frid AH, Kreugel G, Grassi G, et al. New insulin delivery recommendations. Mayo Clin Proc. 2016;91:1231-55.

3. Berger M, Cüppers HJ, Hegner H, Jörgens V, Berchtold P. Absorption kinetics and biologic effects of subcutaneously injected insulin preparations. Diabetes Care. 1982;5:77-91.

4. Bantle JP, Neal L, Frankamp LM. Effects of the anatomical region used for insulin injections on glycemia in type I diabetes subjects. Diabetes Care. 1993;16:1592-7.

5. ter Braak EW, Woodworth JR, Bianchi R, et al. Injection site effects on the pharmacokinetics and glucodynamics of insulin lispro and regular insulin. Diabetes Care. 1996;19:1437-40.

6. Mudaliar SR, Lindberg FA, Joyce M, et al. Insulin aspart (B28 asp-insulin): a fast-acting analog of human insulin: absorption kinetics and action profile compared with regular human insulin in healthy nondiabetic subjects. Diabetes Care. 1999;22:1501-6.

7. Apidra (insulin glulisine). CHMP Assessment Report. Procedure No. EMEA/H/C/557/X/0023. 12 January 2010. Available from: http://www.ema.europa.eu/docs/en_GB/document_library/EPAR_ -_Assessment_Report___Variation/human/000557/WC500090084. pdf. Accessed 19 Jan 2017.

8. Heise T, Hövelmann U, Brøndsted L, Adrian CL, Nosek L, Haahr H. Faster-acting insulin aspart: earlier onset of appearance and greater early pharmacokinetic and pharmacodynamic effects than insulin aspart. Diabetes Obes Metab. 2015;17:682-8.

9. Heise T, Stender-Petersen K, Hövelmann U, et al. Pharmacokinetic and pharmacodynamic properties of faster-acting insulin aspart versus insulin aspart across a clinically relevant dose range in subjects with type 1 diabetes mellitus. Clinical Pharmacokinet. 2016. doi:10.1007/s40262-016-0473-5.

10. Heise T, Pieber TR, Danne T, Erichsen L, Haahr H. A pooled analysis of clinical pharmacology trials investigating the pharmacokinetic and pharmacodynamic characteristics of fast-acting insulin aspart in adults with type 1 diabetes. Clinical Pharmacokinet. 2017. doi:10.1007/s40262-017-0514-8.

11. European Medicines Agency. Committee for Medicinal Products for Human Use. Guideline on the Investigation of Bioequivalence. CPMP/EWP/QWP/1401/98 Rev. 1/Corr. 20 January 2010. Available from: http://www.ema.europa.eu/docs/en_GB/document_ library/Scientific_guideline/2010/01/WC500070039.pdf. Accessed 19 Jan 2017.

12. Food and Drug Administration. Code of Federal Regulations. 21 CFR Part 320. Bioavailability and Bioequivalence Requirements. Available from: http://www.ecfr.gov/cgi-bin/text-idx?SID= 
b77b9e6e4aea3cdbd753c6bbd1b98077\&mc=true \&node $=$ pt21.5. 320\&rgn=div5. Accessed 19 Jan 2017.

13. European Medicines Agency. Committee for Medicinal Products for Human Use. Guideline on Bioanalytical Method Validation. EMEA/CHMP/EWP/192217/2009 Rev. 1 Corr. 2. 21 July 2011.

Available from: http://www.ema.europa.eu/docs/en_GB/document_ library/Scientific_guideline/2011/08/WC500109686.pdf. Accessed 19 Jan 2017.

14. Food and Drug Administration. Center for Drug Evaluation and Research. Center for Veterinary Medicine. Guidance for Industry. Bioanalytical Method Validation. May 2001. Available from: http://www.fda.gov/downloads/Drugs/Guidance/ucm070107.pdf. Accessed 19 Jan 2017.
15. American Diabetes Association. Defining and reporting hypoglycaemia in diabetes: a report from the American Diabetes Association Workgroup on Hypoglycaemia. Diabetes Care. 2005;28:1245-9.

16. Vora JP, Burch A, Peters JR, Owens DR. Relationship between absorption of radiolabeled soluble insulin, subcutaneous blood flow, and anthropometry. Diabetes Care. 1992;15:1484-93.

17. Jain L, Parks MH, Sahajwalla C. Determination of time to onset and rate of action of insulin products: importance and new approaches. J Pharm Sci. 2013;102:271-9. 